\title{
A Study on the Usability Reduction of Mobile Graphic User Interface: In Comparison with De-functionalization Process in Art History
}

\author{
Hye-Joo Lee ${ }^{1}$ and Jong-Hoon Choe ${ }^{2 *}$ \\ PhD Student, Media Interaction Design, Ewha Womans University, Korea ${ }^{1}$ \\ Professor, Media Interaction Design, Ewha Womans University, Korea ${ }^{2}$ \\ bornwater@gmail.com ${ }^{1}$,deadant@ewha.ac.kr ${ }^{2}$
}

\begin{abstract}
The purpose of this study is to examine the changes of a mobile user interface by the emotional influence on the function and usability. I found the reason for its case in the artistic aspect through the art history. In the history of UI (User Interface), the usability has been the key function of UI. Based on usability, the good UI is able to easily find and use the necessary elements and easily obtain the intended result from the element. However, thanks to the technology of semiconductors, digital devices are becoming smaller and lighter, and the development of sensing technology and the appearance of artificial intelligence have changed the role of the UI between users and computers. It has rapidly developed from the time of the universal graphical user interface to the age of natural user interface. The user's interface for the purpose of conversation with the computer changes to a more human and intuitive way of resembling the lifestyle of the person and the purpose of the UI is gradually being shifted from the function center to the emotional center. Furthermore, the NUI (Natural User Interface) is expected to evolve into an OUI (Organic User Interface) that communicates with computers in all its natural state. This reduces the usability of the user interface and maximizes visual sensitivity. By doing so, UI is gradually resembling people and nature.
\end{abstract}

Keywords: UI De-objectification, Segmentation of UI function, Artistic UI, Aesthetic UI, Emotional UI

\section{Introduction}

\subsection{Backgrounds and Research Objectives}

In the history of UI, the most crucial function of UI was its 'Usability'. A disable UI is when the user could find and use the necessary elements based on its usability and obtain the exact results intended from the elements. However, recently some skeptical views have risen about the future of UI featuring on the usability. With the development of semi-conductor technology, digital devices have become smaller and lighter and the progress of sensing technology and AI has brought a massive change in the role of the UI that links the user and computer. From the age of GUI (Graphic User Interface), a rapid change occurred to the age of NUI.

As the user interface changed from communicating with the computer to a more humane, intuitive method that resembles of the human lifestyle, the purpose of the UI existence has also changed to a more emotional than a functional one. Furthermore, it is predicted that the NUI will develop into an OUI where the natural form itself interacts with the computer. Currently, as UI that resembles the human

Received (November 15, 2017), Review Result (January 5, 2018), Accepted (January 18, 2018)

*Corresponding Author 
and nature became an important function, cases of UI with reduced usability and maximized visual sense has increased. [Figure 1]

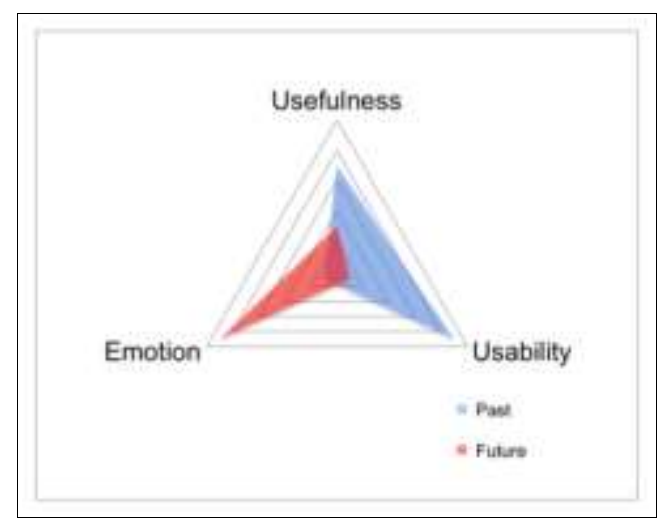

\section{Figure 1. Swift of User Interface Function}

Donald Norman (1935 ), a scholar in design studies mentioned in his book "Emotional Design; Why we love (or hate) everyday things" that the emotional system can change how the cognitive system operates. Thus, emotion and affection have the great impact on the function and usability. Norman found this case in cognitive psychology and former culture and arts.

This study aimed to find the possibility how the role of UI can be changed from usability to aesthetic stimulation in the aspect of how arts, culture, and media evolved and progressed in the era. In the stream of time just as abstract painting does not focus to reproduce objects, I suggested that the function of UI is beyond efficient usability but that the very existence could be an expression of art. Thus it could be expected that the importance of visual UI grounded on emotion and affection will be stressed.

\subsection{Research Method and Area}

The scope of the research was confined to the cultural and the artistic perspectives and changes in technology development were excluded. [Figure 2]

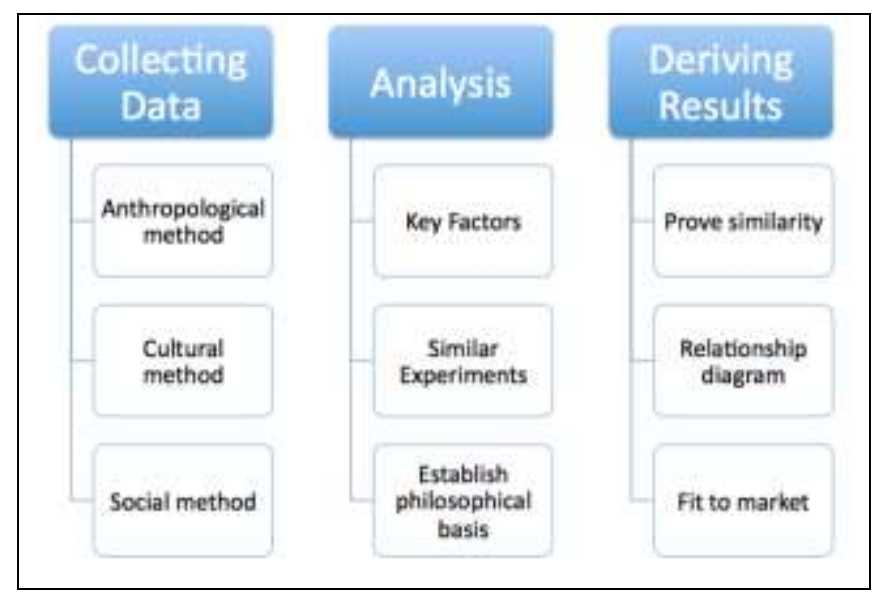

Figure 2. Research Methodology 
In addition, I focused on design with artistic tendency among applications limited to mobile app service, not pure media art using mobile as a tool. The research process was to prove the background in which the aesthetic and emotional UI was born through the two flows of art history.

First, 'De-objectification of the GUI looking from the perspective of conceptual Art' Second, 'Changes in the functionality of the GUI looking from the perspective of the early photography'. The main method of this study was to analyze the above two factors and to derive the characteristics with a similar pattern to the changes of the current UI. With these common points, I could validate the current UI phenomenon and present changes in future UI.

Through this comparative analysis process, I was able to characterize the two changes in the current UI. And the two key factors of them are 'Conceptual GUI' and 'Aesthetic GUI'. And the phenomenon that corresponds to each key factor was explained and proved by each case through the present UI service contents.

\section{Theological Backgrounds and Case Study}

\subsection{De-objectification of the GUI looking from the Perspective of Conceptualism in Art History}

Conceptual art, a branch of postmodernism, is a term written in 1967 when Sol LeWitt(September 9, 1928 - April 8, 2007) published a "paragraphs on conceptual art". It is a new artistic movement that is characterized by one-time, temporal, process, experimental, and de-materialization. It is also an anti-artistic tendency that focuses on ideas and processes of production rather than symbols, photographs, charts, or work themselves, out of the art expressing conventional objects with forms, colors, and materials.

The origin of conceptual art can be found in Marcel Duchamp's 'Ready-mades'. The development of information and communication technology in the 1960s made the importance of the power of information media. In such a socio - cultural atmosphere, art has changed from representation to abstract to another meaning. Such changes raised fundamental questions about the purpose and function of art, raising questions about what art is and what the role of the artist is.

In conclusion, conceptual art is an art that tends to emphasize the non-material aspects of ideality rather than the general material aspects. It abandoned the consciousness of the artist and thought the intention and process of production to be the art itself rather than the work itself. Due to the appearance of conceptual art, sketch, text, and sentence itself were regarded as independent works of art, thus widening the range of art. It enhanced that viewers themselves opened their own imagination through works.

I found that these attempts of conceptual art have much in common with the changing aspects of the current GUI. Among them, I paid more attention that conceptual art redefined the purpose of art and subdivided the fundamental meaning of art from one to several. I also discovered that the evolution of technology at the time that conceptual art could have occurred was similar to the background of the fourth technological revolution, in which the current GUI could change.

Marcel Duchamp (28 July 1887 - 2 October 1968), a representative artist of conceptual art, is a good example of de-objectivity in that he did art opposite to the pure purpose of art at that time. [Figure 3] 


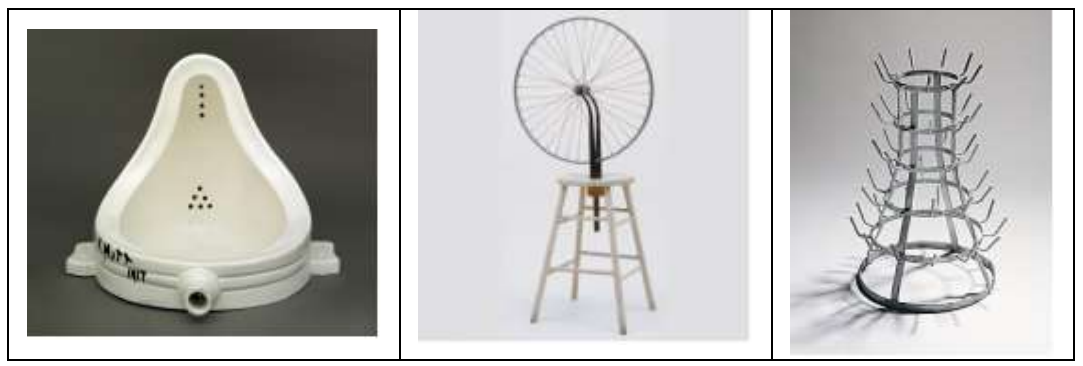

\section{Figure 3. Fonte (1917), Bicycle Wheel (1951), Bottle Rack (1963) by Marcel Duchamp}

Marcel Duchamp (28 July 1887 - 2 October 1968), a representative artist of conceptual art, is a good example of de-objectivity in that he did art opposite to the pure purpose of art at that time. [Figure3] Duchamp's notable features are works using ready-made items. The feature of Ready-made is that it transforms the product that can be bought on the market and it is created as an artwork. It was not a painting, nor a sculpture, but a whole new genre. Such a work completely changed the direction of 20th century art. As a result, the pure painting gradually moved away from the paintings and became more dependent on the viewer's experience. Also, this kind of conceptual art of that age has become a turning point from a plane to a solid.

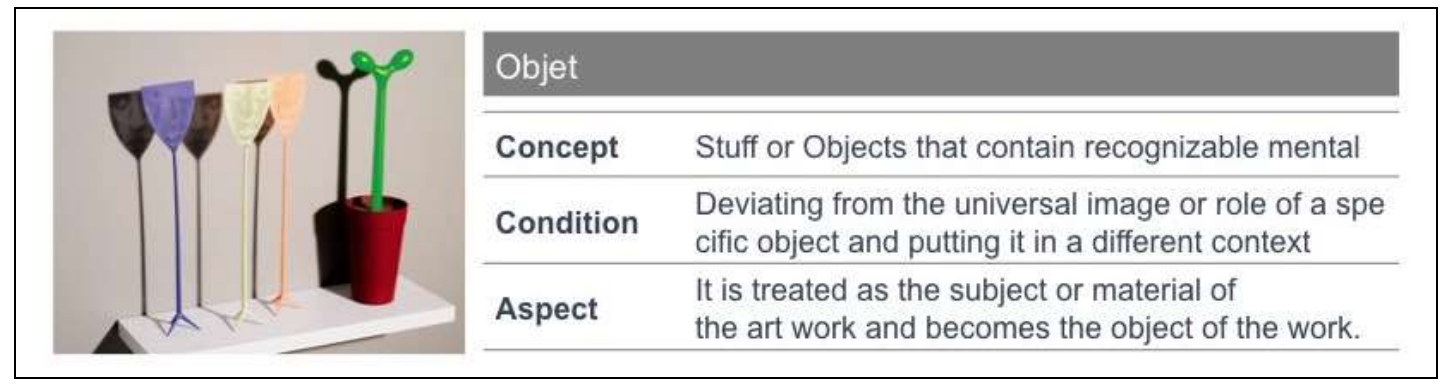

Figure 4. Definition of Objet

Specifically, Duchamp's work entitled 'Fonte' [Figure 3] only with his signature on a mass-produced toilet product had changed into an objet [Figure 4]. At that time, the objet was a precious sculpture so by the ready-mades product turned into an objet, mass-produced products could have a starting point to become a piece of art. In other words, Duchamp's works showed the evidence that the original purpose of arts or products can be changed by the situation of culture and society.

First, the purpose of usage was changed by being given artistry from the original function of the ready-mades product. Second, the main purpose of art at that time was to experience of the beauty of paintings but through conceptual art, the purpose had switched to an experience of the audience. The anti-traditional philosophy of this conceptualism influenced Modernism, Dadaism, Surrealism, and Pop art. [Table 1]

As a result of this phenomenon, in the 1930s, Walter Benjamin (1892 1940, Philosopher) wrote the book "Art in the Age of Technology Reproduction (1936)". His argument is that when technology of one generation has reached saturation, the technology becomes art. This logic is very representative of the current GUI changes. 
Table 1. Art of Ready-made Products by Art History

\begin{tabular}{|l|l|l|}
\hline & Examples & Special feature \\
\hline Dadaism & $\begin{array}{l}\text { Give aesthetic sense to mass-produced } \\
\text { goods or their scrapped products } \\
\text { Transition of meaning }\end{array}$ \\
\hline Surrealism & & \\
& & $\begin{array}{l}\text { Tools to track and develop subconscious } \\
\text { De-functionalization }\end{array}$ \\
\hline Pop Art & & $\begin{array}{l}\text { Artistic development of ready-made } \\
\text { products due to development of } \\
\text { photographic technology } \\
\text { Objet through copying and repetition }\end{array}$ \\
& \\
\hline
\end{tabular}

The most important purpose of GUI was the technology for usefulness and usability but it's its emotional function has recently been emphasized. This can be seen as a process of GUI transforming into an art form. And the art-ification of the ready-mades items shown in the conceptual art are is common evidences found in the past. [Table 2] The segmentation process of art in conceptualism is evidence that the GUI's elements can be segmented and each element can exist as own purpose.

\section{Table 2. Common Point between Conceptual Art and Current GUI Trends}

\begin{tabular}{|c|c|c|}
\hline Name & Conceptual art & Current GUI trend \\
\hline \multicolumn{3}{|l|}{ Example } \\
\hline & $\begin{array}{l}\text { Realism: } \\
\text { The Gileaners(1957) }\end{array}$ & $\begin{array}{l}\text { Existing GUI } \\
\text { with good usability }\end{array}$ \\
\hline \multirow{4}{*}{$\begin{array}{l}\text { Comparison } \\
\text { Analysis }\end{array}$} & Art-ification of Ready-made product & Art-ification of GUI Function \\
\hline & Deviation of role, symbolism & De-objectification of the GUI \\
\hline & Representation and expansion of objects & Maximization of Visuality \\
\hline & Transition of Meaning & Conversion of UI essence \\
\hline
\end{tabular}

The main purpose of GUI is to make users interact with electronic devices with an easy affordable visual indicator. So existing GUI reviewers decided that abstract and obscure GUI has lost its purpose. However, changing the purpose of art through conceptualism suggested that a new GUI trend with emphasis on emotion could change the purpose itself from usability to other new values like play. 


\subsection{Changes in the Functionality of the GUI Looking from the Perspective of the Early Photography}

GUI design refers to the process of expressing abstract concepts, information structures and data of digital products or services in a visual form. That's why the most important function of the GUI is to communicate those concepts and information most effectively. In the early days of HCI, 'Interface Development' was the most important part and the GUI gradually became more important. And now, the usability and usefulness of the GUI functions are most important factors. In other words, GUI was not a process of visual design for beautiful, but it was a process of accurately conveying the value of products and services to users. Therefore, a good interface is not simply a pretty interface, but an interface that faithfully follows the three elements of HCI (Usability, Usefulness, and Affections). [Table 3]

Table 3. Important Features of the Previous UI System

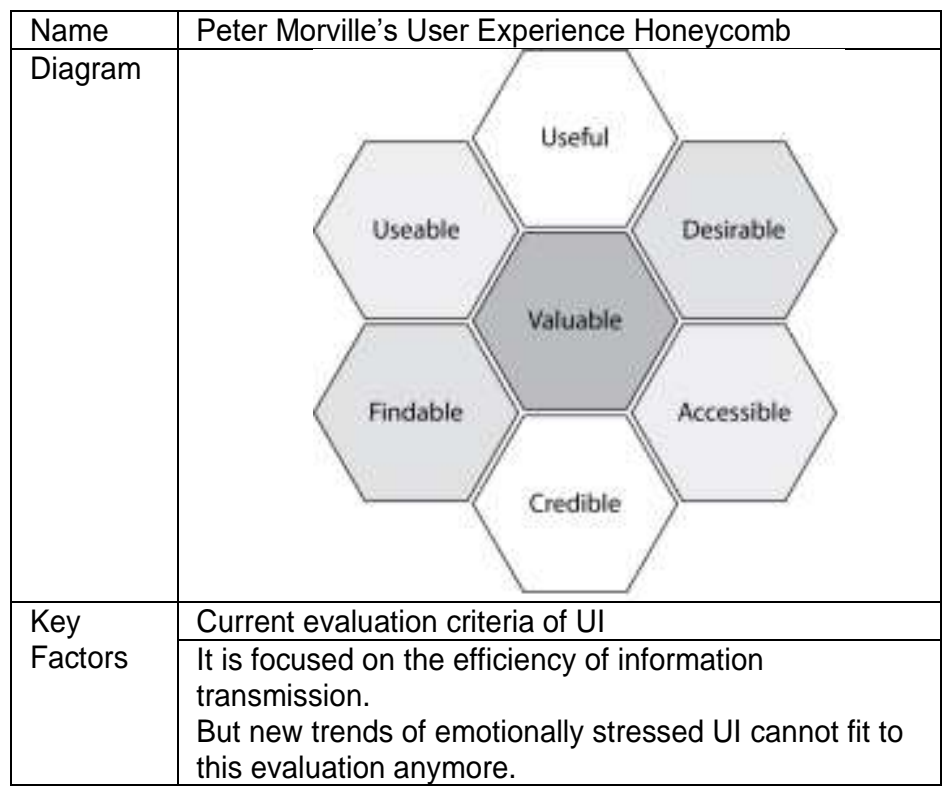

However, looking at the latest GUI trends, the phenomena are reversing. The harmony of the three elements of UI is broken. So, New kinds of GUI has arisen that depend only on the emotion that has a new value function like amusement. So these phenomena are simply evaluated by a misplaced GUI. [Table 4]

These phenomena and evaluations have been repeated and existed in the history of photography. The example was founded in the history of its early photos. The function of the early photographs was to reproduce facts. This is because the beginning of the photo was in the implementation of the technology.

When the function of photography at that time was to change into art, existing artists had criticized the attempt. However, photograph now had became become one genre of arts with never- ending effort. At present, the appearance of a function broken GUI can be explained in the same context as the early art of photography. Walter Benjamin explained the changes of function in early photography in his book "the age of technical reproduction of art has come". His book was the first to describe the relationship between Technology Development and the development of art. 


\section{Table 4. Changes in the Function of GUI by Its Evolution}

\begin{tabular}{|l|l|l|}
\hline Time & Past and Current & Current and Future \\
\hline Name & Affective GUl of Big data & Emotional GUl of Big data \\
\hline Photo & & \\
Example & & \\
& & \\
\hline Diagram & & \\
& & \\
& & \\
& & \\
& & \\
\hline Fnalysis & Focus on usability & Focus on artistic expression \\
\hline Functions & Efficiency & Aesthetics and Amusement \\
\hline
\end{tabular}

His theory explained the emergence of new media and the artistry of technological reproduction, through photography and cinematography. Benjamin's attention here was that the clone reached a stage where it would affect the artistic production itself. Early photographic theorists had argued that the reproducibility of photography was sacrilegious and that only artists had the right to reproduce reality. This view told the artists' fears about not only the provocative emergence of new technologies as an art form but also the technology of reproduction itself. Over the next 100 years, constant changes in photography had created photographs as current art. [Table 5] In the early days of photography, many painters used the silver plate of Daguerre as technical aids.

\section{Table 5. Changes of the Function of Photography by Its Evolution}

\begin{tabular}{|l|l|l|}
\hline Name & Early photo as Technology & Current photo as Art \\
\hline Examples & & \\
& & \\
\hline Title & $\begin{array}{l}\text { Dorothy Catherine Draper } \\
\text { by John William Draper 1839 }\end{array}$ & $\begin{array}{l}\text { NO title by: Monty } \\
\text { Knowles } \\
2014\end{array}$ \\
\hline Function & Reproduction of facts & $\begin{array}{l}\text { Visualization } \\
\text { unconsciousness }\end{array}$ \\
\hline
\end{tabular}

However, in the photographs, artists had come to find visual irrefutable unconsciousness. The nature of the 'camera' device was that it had a different visual representation than the nature of the eye. The reason was that human consciousness projected their consciousness by the way of photography (perspective, enlargement, reduction, contrast etc). Benjamin named it "the world of visual unconsciousness" in his book (in Technique Replica Art II, Chapter 16, Chapter 3, Chapter 13). [Table 6] 


\section{Table 6. Comparison of Photography as a Technology and as an Art}

\begin{tabular}{|l|l|l|}
\hline Name & Reproduction of facts & Artistic Expression \\
\hline Examples & & \\
Title & $\begin{array}{l}\text { Searched by Google } \\
\text { image }\end{array}$ & $\begin{array}{l}\text { Pepper NO.30, Edward Western, } \\
1930\end{array}$ \\
\hline Analysis & $\begin{array}{l}\text { Human consciousness can project their consciousness by the } \\
\text { way of photography like perspective, enlargement, reduction, } \\
\text { contrast etc., }\end{array}$ \\
\hline
\end{tabular}

\section{Analysis of GUI Design with Reduced Usability}

\subsection{Examples of Conceptual GUI}

A conceptual GUI means the UI is characterized by De-objectification and subdivision of essence. According to the background of II.1, conceptual GUIs are often seen in current UI services. 'Tamagotchi Gestures' is an example that illustrates this conceptual GUI well. From the article "Tamagotchi Gestures and UX Design" by Samantha Silver (UX Researcher, Key Lime Interactive), Tamagotchi [Figure 5] is small, keychain virtual pet toy of the late 1990s and early 2000s. Tamagotchi was essentially a small egg-shaped computer with an interface usually consisting of three buttons and inside this small device lived a little creature that users would care for from it's birth to its death. By 2010, this simple play was sold 76 million worldwide people.

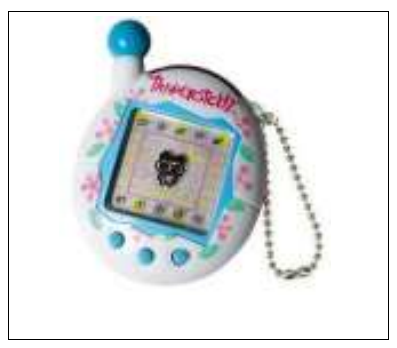

Figure 5. Tamagotchi

The UI design of the Tamagotchi that made it so popular was very easy and simple. New needs of users like simplicity arose when users are sick of overwhelming data and complexity of mobile contents and services. And today, the technology of sensors and AI could make mobile devices easier and simper. The Tamagotchi Gesture brought a much-needed human and emotive touch to the world where efficiency and dispensability reign supreme.

Today, users expected new function when they use mobile devices. And this need changed the purpose of UI and devised the functions to the new categories. Chase Buckley, a Design Evangelist commented, "More and more product designers are deliberately invoking the Tamagotchi Gesture in their work - crafting personality 
and charm by imbuing their products with a certain obsolescence, incompleteness, and fragility." The idea of implementing Tamagotchi Gestures into UI design may not be the most efficient or practical in terms of usability, but in turn, gives a certain charm or playful experience. The balance in between usability and user experience could be broken in this UI design. However, as in the case of conventional conceptual arts, this phenomenon can also be explained as a subdivision of UI with a different purpose.

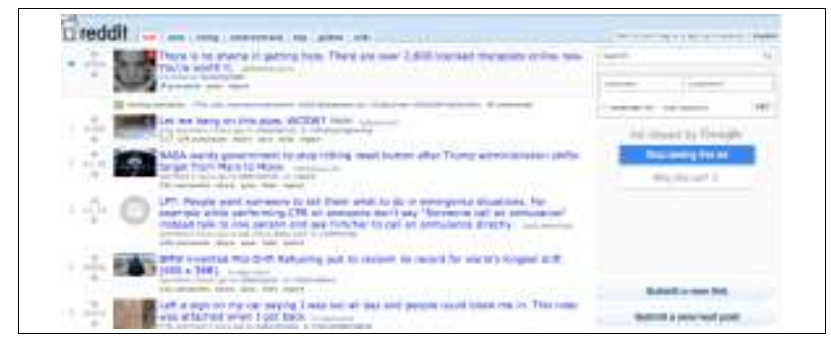

Figure 6. Online Forum 'Reddit'

Samantha Silver explained online forum 'Reddit' [Figure 6] as a good example of Tamagotchi Gesture. Their user interface is not ideal in terms of usability because it's bare-bones design and forum-style organization makes the website itself difficult to new users.

However, Reddit was extremely popular and successful. It provided a positive user experience for their uniqueness and kept people coming back and this was the main characteristic of the Tamagotchi Gesture. William Gibson, author of Wired Magazine stated this phenomenon that they're pointless yet needful, comforting precisely because they require tending. Thus, new needs like humanity or a sense of comfort made by lack could create a conceptual GUI. Conceptual GUI like Tamagotchi Gesture can invoke a sense of originality, emotion, and charisma into mobile devices that will ultimately enhance the overall user experience.

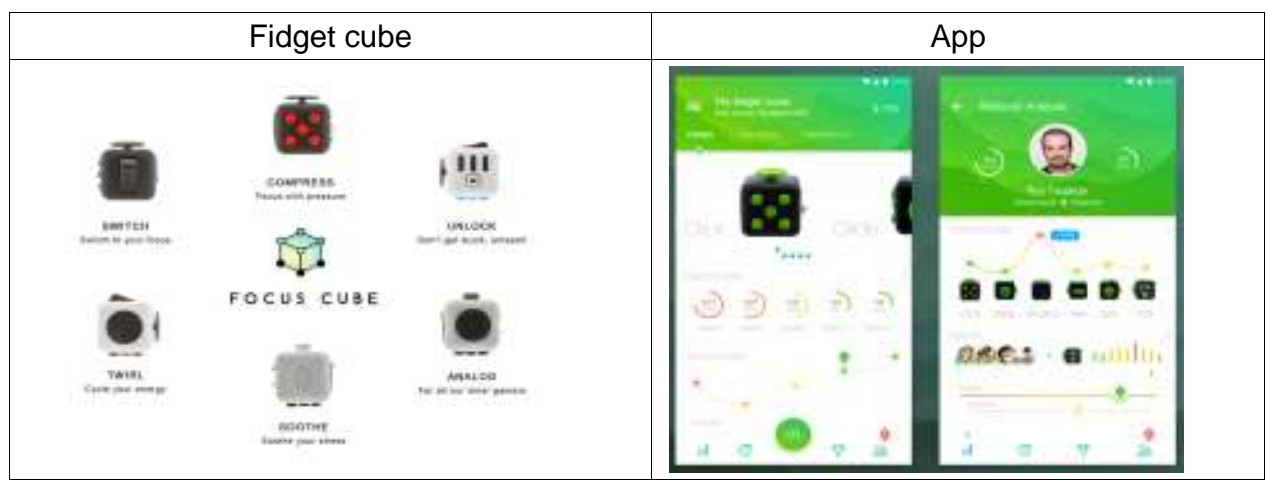

Figure 7. Fidget Cube App

Another example of a conceptual GUI is a 'Fidget Cube App' [Figure 7] that represents the emergence of new subdivisions of functionality. Fidget Cube is a desk toy for anyone who likes to fidget. It has six sides of buttons (click, glide, flip, breath, roll, and spin). These buttons are originally used as a button of mobile devices as a Physical User Interface. But The PUI was separated from the mobile device and made a new feature with the new value. The value meant user's new needs such as stress reduction, simple joy, or a meditation. The Fidget Cube is separated from the device and used as a toy object, but the data that touches the object is analyzed and stored through the mobile app. The app tracks user's behavior 
while using the cube and daily analyses is based on the action and frequencies. With the data, it gave users a full report of stress levels in various scenarios and locations. It can also suggest a medical advice if detects high levels of stress and anxiety. The user can also share their mental status with friends and family.

This simple Toy object does not have any efficiency or usefulness. It seems it only represent simple joy or the habits, which is annoying, but users created the new functions like boosting attention or memory through playing Fidget Cube. Researchers (Katherine Isbister, Michael Karlesky) at the Polytechnic Institute of New York University commented that minor distractions can help boost productivity by giving the mind a break, making it easier to pay attention to the task upon returning to it. But a key thing that separates a fidget widget from other distractions is that it's used for the enjoyment of the experience itself not to achieve any particular goal. They also theorize that fidget widgets are playful, secondary interactions able to engage the interrelation of bodily movement, affective state, and cognition to support primary serious tasks.

This phenomenon meant that even though the elements of the existing GUI are overturned and the previous balance is broken, separated elements could perform full functions in their respective areas for a new purpose.

\subsection{Examples of Aesthetic GUI}

As analyzed in 2.2, the graphical elements of the GUI were highlighted as rising demands of its emotional function. According to "UI Design trends 2017" by Aaron Gustafson (web standards \& accessibility advocate at Microsoft), Animated graphics of GUI will be more extended. The technical changes like HTML5 can make GUI graphics more widely animated. As UI designs moved more for the flat design patterns, animations also became faster, smoother and nicer. Visual extended GUI design is used as a great way to quickly grab the user's attention. Because vision is the strongest of all human senses and a large, single image is able to summarize both message and tone of voice in an efficient, succinct way. Aaron Gustafson forecasted interactive 3D graphics would be used as a means of engagement and storytelling.

In addition, users living in the mass-information age are increasingly demanding to escape from the existing GUI framework, which focuses on information transmission only. Therefore, the new tendency is created to feel human nostalgia through GUI, which is close to the art such as illustration. It meant that nowadays, users are trying to get the experience of beauty through the real mobile service.

'Haeckel Clock' [Figure 7] by Julapy (Interactive design company, by Lukasz Karluk) is a perfect example of esthetic UI with visual emphasis. In Lukasz Karluk(Digital artist in Julapy) 's explanations, Haeckel clock is an interactive clock app for the iPad inspired by the work of Ernst Haeckel(1834 1919, Biologist). The clock elements that represent the time are based on radiolarians, a group of single- celled organisms, which were often the focus of Haeckel's studies and his intricate illustrations. The clock shows the time by user's toggle like double tapping on the screen. It arranges the units of time into a circular nucleus where the digits of the current time are pulled into the center while the unused digits orbit around the nucleus. As the second's tick by the digits rearranges itself in a kind of evolutionary dance creating a unique and generative representation of time, each digit is interactive so users can grab it and flick it around which makes a fun tactile experience. The iPad accelerometer is also used to add extra motion to the digits by swaying them in the tilt direction. Even if it fails to read the clock efficiently, the App based on this artistry explains the artistic process of the UI well. 


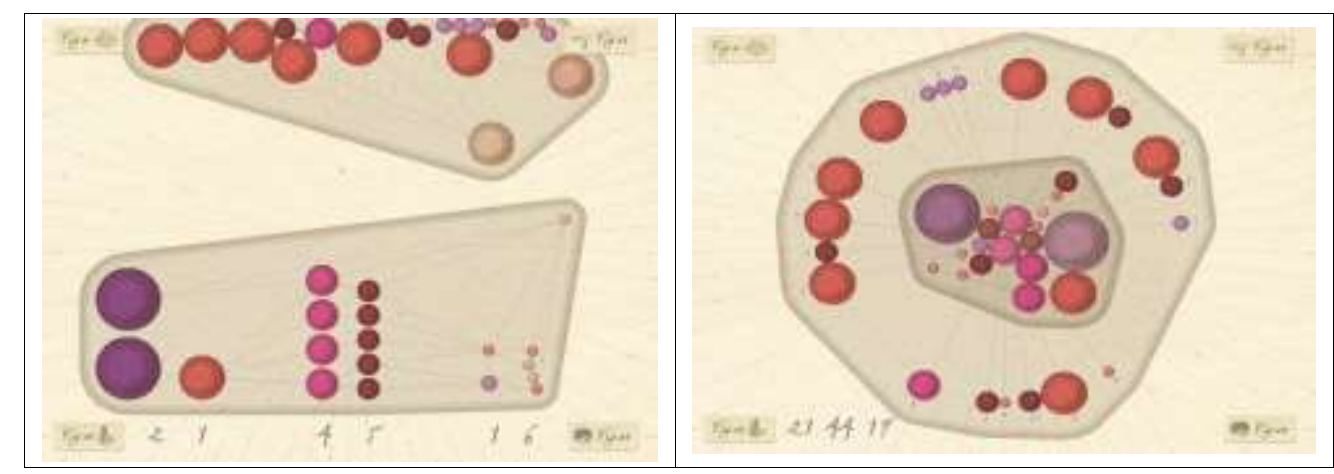

Figure 7. Haeckel Clock

Another example, the big data simulation App 'Data Vis' is completely destroyed the usability of the UI and exists as the form of a graphic art. [Figure 8] So it is both an esthetic GUI and an interactive media art. Data Vis is a real-time generative visualizationdriven by seismic data, created for a Multi- National Energy Company. Code on Canvas (Founded by Lukasz Karluk and René Christen) produced a real-time generative application, which visualized seismic data. The animation made use of the simple, yet powerful point-cloud aesthetic to abstract the data and make for interesting viewing. In a big data design, using a statistic graphs or charts are no longer a solution. At some point, data visualization can be a good solution just to feel and look the whole view of the big data. Also, the visualized image itself is very aesthetic and has a strong artistic power.

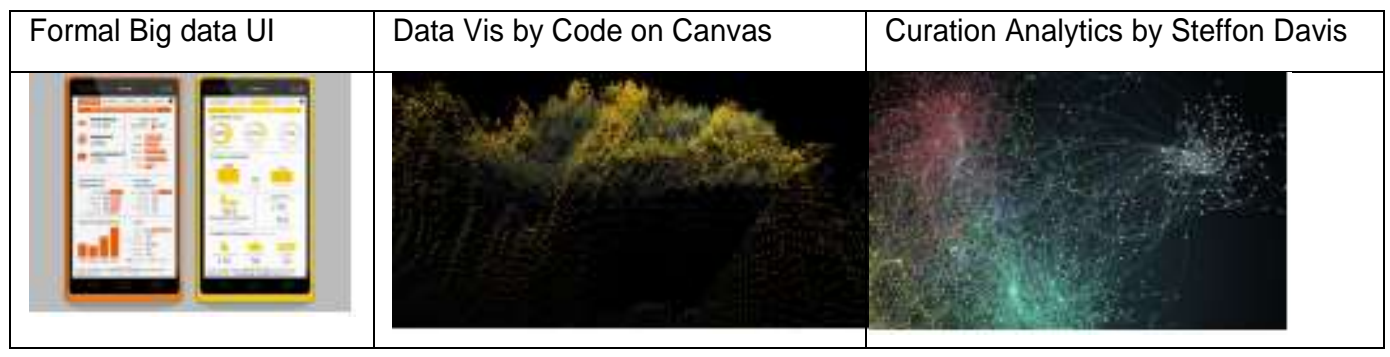

Figure 8. Big Data Visualization

According to a dissertation of "A Comparative Study about Style Change of Modern Art and GUI"(Kang-E Lee, Jong-Hoon Choe), GUI is currently in the initial stage of abstraction. Describing it in Deeper theoretically, the aesthetic UIs that are presently being reduced in usability can be described by Immanuel Kant (Philosopher, 1724 1804) as the aesthetic nature of the human being like 'the purpose without purpose'. In other words, the more human emotion is contained in the UI, the more the UI judgment standard had to follow the aesthetic judgment. This already means that the UI will have a value judgment that can be perceived differently by the users who are not objective, formal, and has no purpose or justification.

\section{Conclusions}

This research is meaningful because the emotion-enhancing GUI caused by the imbalance of the basic elements of the UI should not be evaluated as a misplaced UI but should be evaluated as another UI development from the artistic point of view. I have noticed that the expectations, needs, attributes, and user environment of the user, which is the key axis of UX, are changing due to the rapid evolution of technologies such as sensing, artificial intelligence, and html5. Users were already 
generating new needs, such as simple pleasures, relaxation, stress relief, or humanity. Such needs were changing the basic functionality and purpose of the UI.

I have noticed the process of GUI becoming more like a human being by creating new categories like NUI and OUI. The process of humanization of the GUI has brought emotional enhancement, which has been implemented as segmentation of function and visual emphasis.

Through this research, I have found that the process of de-objectification, segmentation, and artistry shown in current GUI trends were shown same in the history of art. As a result, I could prove the existence value of the artistic UI with reduced usability and predicted that the UI that depended on emotion will continue to appear in the future. Also, in recent future, it is expected that a 'Usability Evaluation Method' suitable for UI that is specialized for emotion is also needed. Based on the results of this paper, I will research and develop mobile device service contents based on emotional oriented application GUI as a follow- up study.

\section{Acknowledgments}

This paper is a revised and expanded version of a paper entitled [GUI Changes of Digital Application with Aesthetic] presented at [The 14th 2017 International Interdisciplinary Workshop Series, The 5th International Conference on Interdisciplinary Research Theory and Technology, IRTT 2017), Daejoen, 12. 21 23. 2017].

This research was partially supported by a National Research Foundation of Korea Grant funded by the Korean Government (NRF-2017-Global Ph.D. Fellowship Program).

\section{References}

[1] H. J. Lee and J. H. Choe, "GUI Changes of Digital Application with Aesthetic Emphasis; Limited to the Mobile App GUI with the Characteristics of Media Art", International Journal of Art and Culture Technology, vol. 1, no.2, (2017), pp. 19-24.

[2] K.-E. Lee and J.-H. Choe, "A Comparative Study about Style Change of Modern Art and GUI", International Journal of Applied Engineering Research, vol. 11, no 2, (2016), pp. 788-792.

[3] D. Norman, Editor, "Emotional Design", The perseus books Publishers, NewYork, (2004).

[4] Kim Jin Woo, Editor, "Introduction of HCI", Ahn Graphics Publishers, Korea, (2012).

[5] Y. J. Lee, Editor, "Theory and Practice of UI/UX", Hanbit academy, Publishers, Korea, (2015).

[6] K. M. Gu, Editor, "4th Industrial revolution business trends", Jungbo Munwha, Publishers, Korea, (2017).

[7] W. Benjamin, "The Work of Art in the Age of Mechanical Reproduction", Edited Hannah Arendt, Illuminations Publishers, London, Fontana, (1968), pp. 214-18.

[8] W. Benjamin, "On the Small History of Photography", Edited Walter Benjamin, Gesammelte Schriften Publishers, Frankfurt, vol. II, (1977), pp. 368-385.

[9] W. Benjamin, "Parisian Letters, II. Painting and Photography", Edited Walter Benjamin, Collected Writings, Frankfurt, vol. III, (1981), pp. 495-507.

[10] M. Karlesky and K. Isbister, "Designing for the physical margins of digital workspaces: fidget widgets in support of productivity and creativity", Proceedings of the 8th International Conference on Tangible, Munich, Germany, (2014).

[11] Keylimeinteractive, http://blog.keylimeinteractive.com/tamagotchi-gestures-and-ux-design, (2017).

[12] Medium, https://medium.com/@ChaseBuckleyUX, (2017).

[13] Namu Wiki, https://namu.wiki/w/reddit, (2017).

[14] Dribble, https://dribbble.com/shots/3402307-UI-Fidget-cube-behaviour-analysis-and-social-sharing, (2017).

[15] Kickstarter, https://www.kickstarter.com/projects/antsylabs/fidget-cube-a-vinyl-desk-toy, (2017).

[16] Behance, https://www.behance.net/gallery/50978075/Fidget-cube-behaviour-analysis-and-socialsharing, (2017).

[17] Codeoncanvas, http://codeoncanvas.cc/, (2017).

[18] Julapy, http://www.julapy.com/, (2017). 